\title{
Fuzzy-based Leader Selection for Topology Controlled PEGASIS Protocol for Lifetime Enhancement in Wireless Sensor Network
}

\author{
Vibha Nehra, Raju Pal, Ajay K Sharma \\ sharda University, Greater Noida, UP \\ vibha.nehra@sharda.ac.in \\ Jaypee Institute of Information Technology, Noida, UP \\ raju3131.pal@gmail.com \\ Dr. B R Ambedkar NIT, Jalandhar, Punjab \\ sharmaajayk@nitj.ac.in
}

\begin{abstract}
Wireless sensor Networks present a new generation of real time embedded systems with limited computation, energy and memory resources that are being used in wide variety of applications where traditional networking infrastructure is practically infeasible. Legitimate leader selection can drastically improve the lifetime of the sensor network. This paper proposes fuzzy logic methodology for leader election in PEGASIS based protocol PEGASIS-TC [1, 17] based on two descriptors- residual energy of node and its proximity to Base Station. Simulation results show that depending on network configuration, a substantial increase in stability of network lifetime can be accomplished as compared to PEGASIS-TC.
\end{abstract}

\section{General Terms}

Topology control, fuzzy based leader selection, PEGASIS.

\section{Indexing terms}

Topology controlled PEGASIS, Fuzzy based leader selection, lifetime enhancement, wireless sensor network.

\section{Academic Discipline And Sub-Disciplines}

Real time embedded system, wireless sensor network.

\section{SUBJECT CLASSIFICATION}

Wireless Sensor Network.

\section{COVERAGE}

Topology control in PEGASIS protocol for WSN.

\section{TYPE (METHOD/APPROACH)}

Experimental- made a virtual model of sensor networks and simulated using MATLAB.

\section{INTRODUCTION}

With advent of tight coupling of conventional personal wireless communication network with physical world, Wireless sensor networks are attaining focus on applications. Applications of wireless sensor network include monitoring of harsh inhospitable, remote geographical locations like toxic urban industrial locations or a surveillance field. Other applications may include office automation, robot control, smart homes, interactive toys, identification and personalization [2]. WSN consists of resource constrained nodes called sensors, deployed for collecting information from sensor field and transmitting the gathered data to a distant Base Station [3, 4]. Lifetime of Wireless Sensor Network may be defined as the time from the inception to the time when network becomes non-functional. A Network may become non-functional when a single node dies or when a particular percentage of nodes perish depending on application [5]. It is universally acknowledged that balanced energy dissipation for equalizing residual energy of nodes is one of the keys for extending network lifetime. Therefore, designing energy efficient protocols is important in the domain of data routing $[5,6]$.

Various efforts have been done in WSNs to implement the fuzzy logic control for legitimate leader selection so as to balance the energy dissipation among the nodes. Mainly leader selection has been done based on descriptors like energy [7- 13], local distance [8,10,11, 12, 13], centrality of node in a cluster [7, 10], quantity of nodes in transmission range [7, $9,12]$. Intelligent techniques have variegated applications in wireless networks [14]. Fuzzy logic control is capable of making real time decisions, even with incomplete information. Conventional control systems rely on an accurate representation of the environment, which generally does not exist in reality. Fuzzy logic systems, which can manipulate the linguistic rules in a natural way, are hence suitable in this respect. Moreover it can be used for context by blending different parameters - rules combined together to produce the suitable result.

PEGASIS is a chain based protocol [17] which uses greedy approach for chain formation which leads to the formation of long links. Moreover, Energy consumption in leader node for transmission of data to BS is the main problem as it consumes significant amount of energy. PEGASIS-TC $[1,17]$ uses the concept of topology control for chain construction which exploits spatial node redundancy and hence conserves energy which can be used in later stages to increase the 
network lifetime. In this paper, we use Topology control for exploiting spatial node redundancy in chain formation and fuzzy logic with descriptors namely, residual energy of node and its proximity to BS for election of leader. The simulation results show that F-PEGASIS-TC outperforms PEGASIS-TC.

The rest of the paper is organized as follows: Section II explains radio energy dissipation model, network model and assumptions of the system. Section III describes the algorithm F-PEGASIS-TC. Next section gives an overview of Fuzzy Inference System. Section V describes PEGASIS-TC-FL system model for leader selection. Section VI lists the performance metrics used for the simulation which gives the results shown in section VII. Section VIII concludes the analysis.

\section{RADIO ENERGY DISSIPATION MODEL}

In the radio energy model $[15,16]$, the transmitter dissipates energy to run the radio electronics and the power amplifier, and the receiver dissipates energy to run the radio electronics as shown in Figure 1.

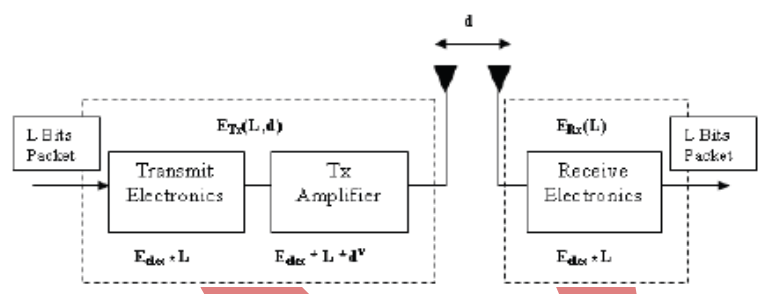

Fig 1. Radio Energy Dissipation Model

There are two different radio models proposed: The free space model and the multi-path fading channel model. When the distance between the transmitter and the receiver is less than threshold value $d_{0}$, the algorithm adopts the free space model ( $d^{2}$ power loss). Otherwise, the algorithm adopts the multi-path fading channel model ( $d^{4}$ power loss). So, if the transmitter sends I-bit message to the receiver up to a distance of $d$, the energy consumption of the transmitter and receiver can be calculated by the following equations:

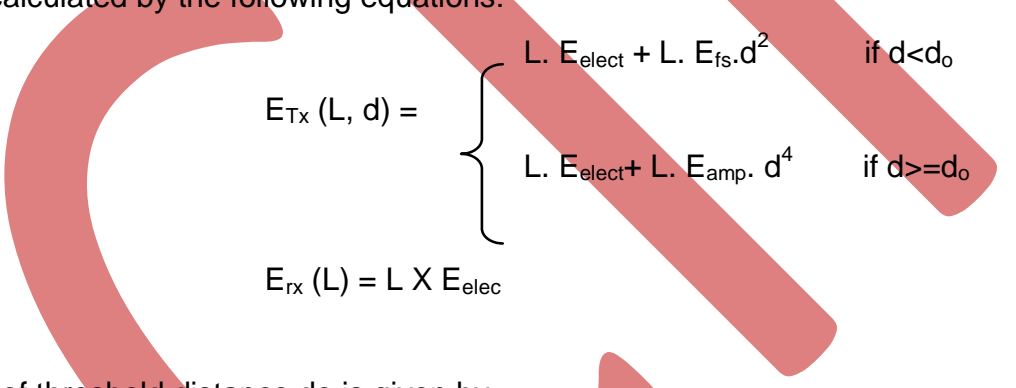

Where the value of threshold distance do is given by

$$
\mathrm{d}_{0}=\sqrt{\frac{E_{f s}}{E_{m p}}}
$$

Our sensor network consists of 100 nodes in a $100 \times 100$ sensor field as shown in Figure 2.

For simplicity, we have taken following assumptions $[4,18]$ :

- All nodes are static.

- All nodes have power control capabilities, and each node can change the power level and communicate with BS directly.

- BS is located far away from the sensor field and at a fixed location.

- For a given signal to noise ratio, symmetric radio channel, making the energy required to transmit from one point to another and in reverse direction identical.

- Nodes always have data to send.

- Every sensor node generates a fixed size packet and forwards it to next node in the chain.

- BS schedules transmission based on TDMA to avoid collision. 


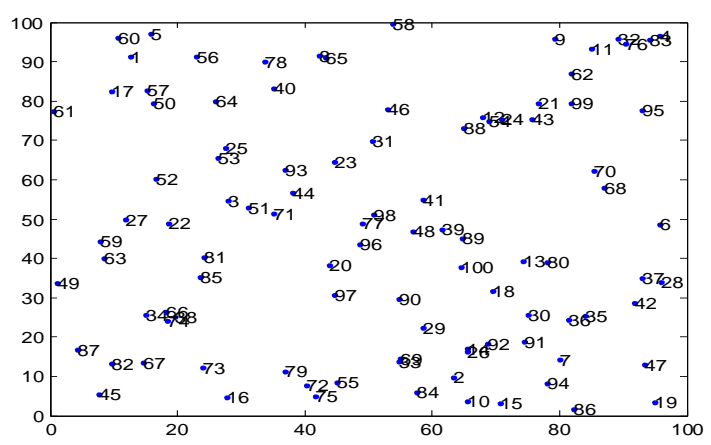

Fig 2. Random Wireless sensor network

\section{F-PEGASIS-TC}

F-PEGASIS-TC is an improved chain based routing algorithm based on PEGASIS-TC [1, 17] which operates in rounds. Each round consists of 3 stages: (1) Chain Construction, (2) Leader Selection phase, (3) data transmission phase. Here, chain construction is same as that in PEGASIS-TC.

\subsection{Chain construction phase}

The algorithm uses the following steps to form a chain:

a) Initialize the network parameters. Determine the number of nodes, initial energy, BS location information et al. Then, the chain construction starts.

b) BS broadcasts the whole network a hello message to obtain basic network information such as ID of nodes alive, distance of each node to BS, and distance among the nodes.

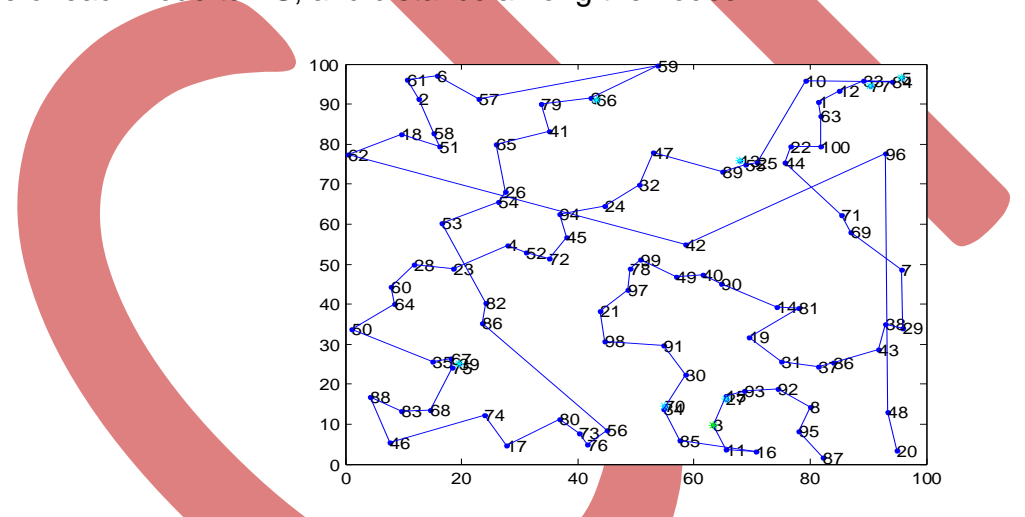

Fig 3. The chain formed in PEGASIS-TC

C) Set the node farthest from BS as end node, it joins the chain first and is labeled node 1.

d) Calculate $\mathrm{C}_{\mathrm{TR}}$ as follows:

i. Sort all the distances between the nodes of the sensor field.

ii. Select $1 \%$ of minimum distances

iii. Compute their average as AVG

iv. Set $\mathrm{C}_{\mathrm{TR}}$ as $3 / 8^{*} \mathrm{AVG}$

e) Find the nodes which are at distance $=<\mathrm{C}_{T R}$ from end node.

f) Turn off the selected nodes.

g) Find minimum distant active node from end node.

h) Join it to chain \& make it next end node.

i) Continue steps e) to $h$ ) till all the nodes have been traversed.

The chain building scenario in PEGASIS-TC for a network of 100 nodes randomly arranged is shown in figure 3.

\subsection{Leader selection phase}


The leader selection in F-PEGASIS-TC uses Fuzzy logic, which smartly assigns leader to nodes which can efficiently utilize the available energy resource. Fuzzy Inference system has been introduced in section IV and later system model has been explained in Section V.

\subsection{Data transmission phase}

Data transmission starts on successful construction of chain and leader node selection. Leader node initiates a token passing approach to start data transmission till the ends of the chain. Each node delivers its own sensed data to its neighbor node in the chain during their time slots assigned by TDMA mechanism. Then, the neighbor nodes fuse the received data with their own data \& forwards further towards the leader. One round will end until BS receives data from the leader. The sleeping nodes are activated and added to the chain as soon as nodes in $\mathrm{C}_{\mathrm{TR}}$ are drained out. In addition, it is assumed that chain is rebuilt when a node of the chain dies during simulation of experiment.

\section{FUZZY INFERENCE SYSTEM}

The FIS has four parts and the architecture of the model is depicted in figure 4 [19].

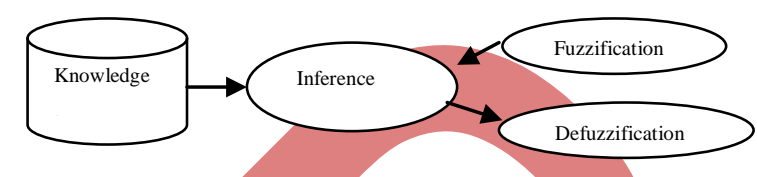

Fig. 4 FIS Architecture

- Fuzzification module: transforms the system inputs, which are crisp numbers, into fuzzy sets. This is done by applying a Fuzzification function.

- Knowledge Base: stores IF-THEN rules.

- Inference engine: simulates the human reasoning process by making fuzzy inference on the inputs and IF-THEN rules.

- Defuzzification module: transforms the fuzzy set obtained by the inference engine into a crisp value.

We use Mamdani method [19] for the inference process of the proposed method. Mamdani's method is used most commonly in different applications, due to its simple structure of 'min-max' operations. The crisp values are obtained in four steps from a FIS system. First, evaluate the antecedents for each rule. Second, obtain each conclusion for each rule. Third, aggregate conclusions and finally, Defuzzification

\section{PEGASIS-TC-FLSYSTEM MODEL}

In the proposed system, we assume that nodes of wireless sensor network can get their coordinate. The PEGASIS-TC-FL system has three parts, three Fuzzification functions, an inference engine (concludes 25 rules) and a Defuzzification module. The architecture of the model is shown in figure 5 [20], but Defuzzification module is not shown in the figure because it's just a formula and has been shown later.

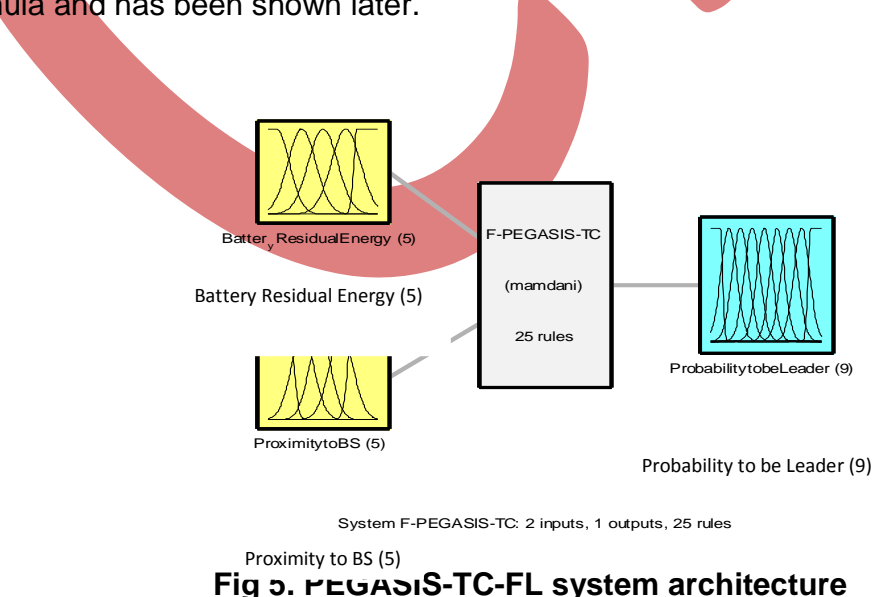

\subsection{Fuzzification module}

Fig o. rEGADIS-TC-FL system architecture

We assume that two attributes of the nodes are required to devise the leader selection. Two input functions are used to transform the systems inputs into fuzzy sets namely, residual energy of node and proximity of a node to the base station. Each input function has five membership functions that depict the degree of the membership function and also illustrate the relationship among the input functions is more numerical. Table 1 shows the input functions.

Table 1: Input functions

\begin{tabular}{l|ll} 
Input & Membership
\end{tabular}




\begin{tabular}{|l|l|l|l|l|l|}
\hline Residual Energy & Very Low(0) & Low (1) & Medium(2) & High (3) & Very High (4) \\
\hline Proximity to BS & Very Near (0) & Near (1) & Medium (2) & Far (3) & Very Far (4) \\
\hline
\end{tabular}

The output function is consists of 9 membership functions. Higher number of membership functions is used because the differences of importance of the inputs can be reflected more clearly $[9,21]$. Table 2 depicts the output functions. The three Fuzzification functions (residual energy, proximity to BS, and probability of being leader) are shown in figure 6.

\section{Table 2: Output Functions}

\begin{tabular}{|c|llll|}
\hline Output & \multicolumn{3}{|c|}{ Membership function } \\
\hline Probability of being leader & $\begin{array}{l}\text { Very Weak (0) Weak(1) Little weak(2) Lower medium(3) Medium(4) Higher } \\
\text { medium(5) Little strong(6) Strong(7) Very strong(8) }\end{array}$ \\
\hline
\end{tabular}

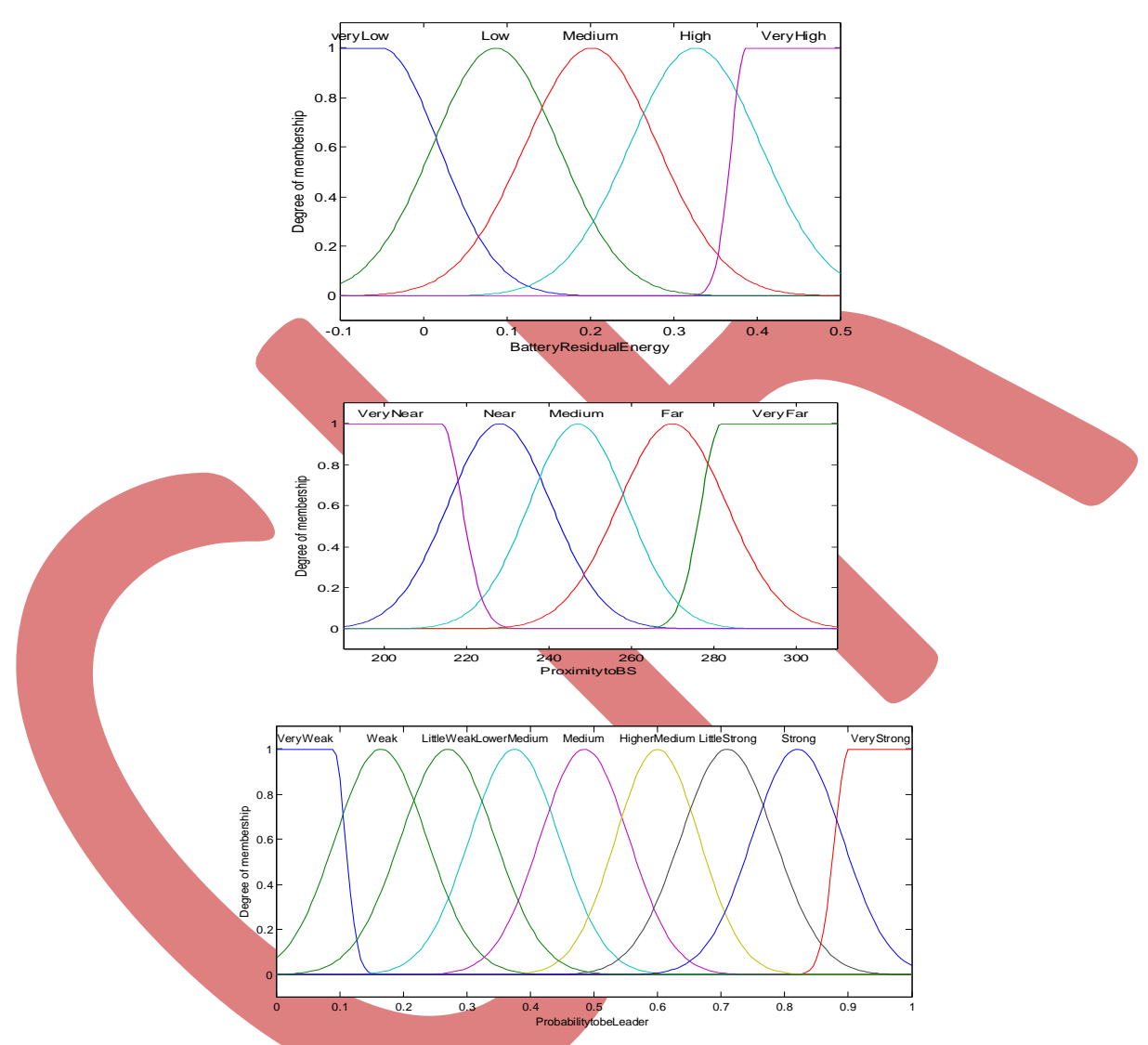

Figure 6. Fuzzification functions: (a) Residual energy (b) Proximity to BS (c) Probability of being leader

\subsection{Knowledge base}

Our system consists of 25 rules in the fuzzy inference. The form of the rules is: IF A and B THEN C, where A, B, C represent battery residual energy, proximity to BS and Probability of being leader. The rules are shown in Table 3.

Table 3. Rules

\begin{tabular}{|l|l|l|}
\hline $\begin{array}{l}\text { Residual } \\
\text { Energy }\end{array}$ & $\begin{array}{l}\text { Proximity to } \\
\text { BS }\end{array}$ & Probability \\
\hline Very Low & Very Near & Weak \\
\hline Very Low & Near & Weak \\
\hline Very Low & Medium & Weak \\
\hline Very Low & Far & Very Weak \\
\hline Very Low & Very Far & Very Weak \\
\hline Low & Very Near & Lower medium \\
\hline
\end{tabular}




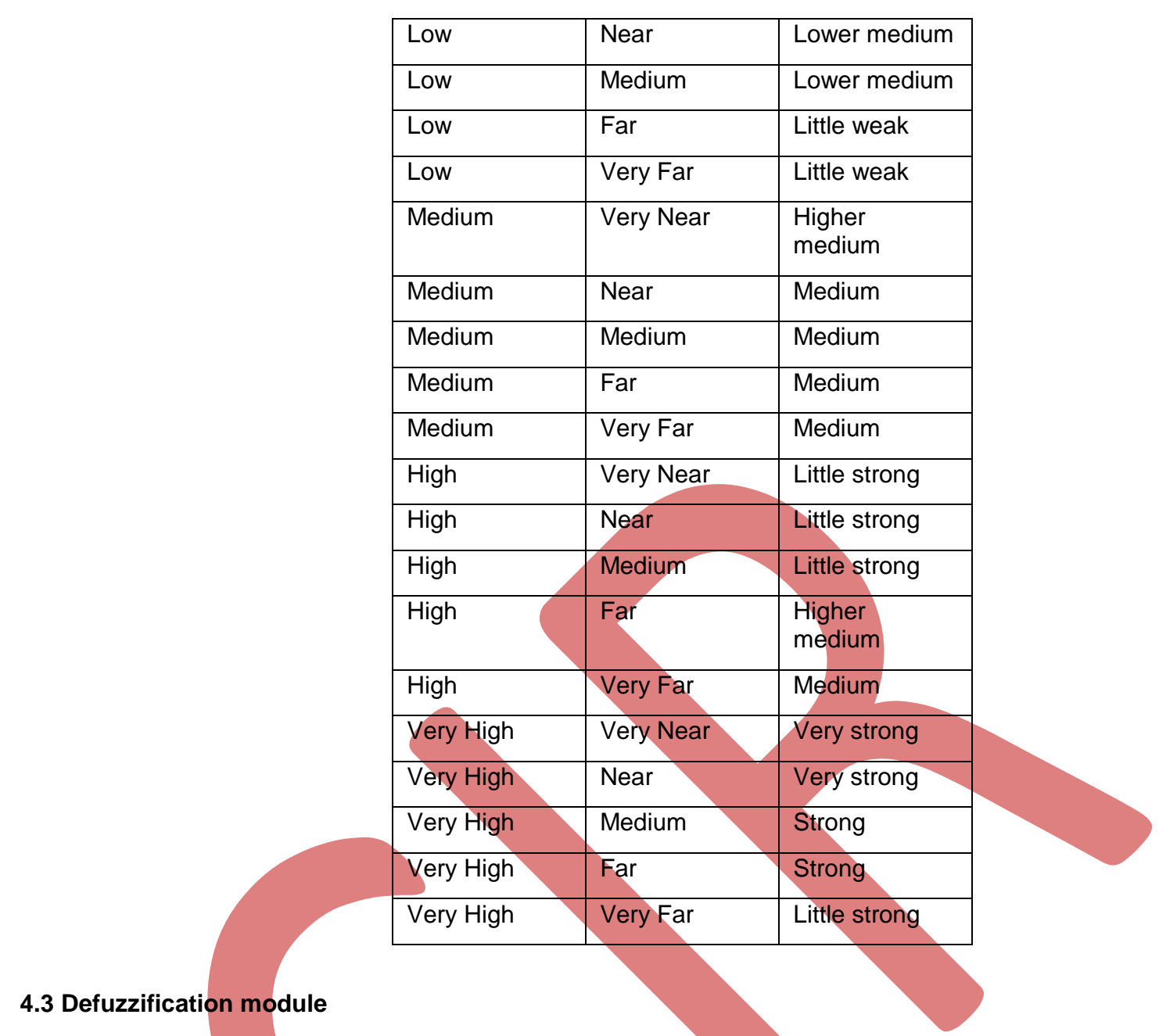

A Defuzzification method is required to obtain crisp values after aggregating the conclusions from each rule. One of the most accepted Defuzzification methods is the Centroid, which returns the centre of the area under the fuzzy set obtained from aggregation of conclusions. We use formula $\mathrm{G}(\mathrm{i})$ to get the value of the probability:

$$
\mathrm{G}(\mathrm{i})=\frac{\sum_{j=1}^{n} X_{j} \cdot u\left(X_{j}\right)}{\sum_{j=1}^{n} u\left(X_{j}\right)}
$$

Each node of the WSN calculates a value G(i) through the above system. Every node gets a random number between 0 and 1. All nodes are arranged on the basis of probabilities; the node with the highest probability is elected as leader for that round.

\section{PERFORMANCE METRICS}

The number of Nodes Alive, number of Packets received at BS, Energy consumed per round \& Total Residual Energy of the sensor network are the performance parameters that have been used to study and evaluate the performance of the proposed protocol.

\subsection{Number of alive nodes}

This instantaneous measure reflects the total number of nodes and that of each type that has not yet expended all of their energy.

\subsection{Data packets received at base station}

It is total number of data packets or messages that are received by the base station. This is also a measure of amount of information sent to BS from the sensor field. This measure varies linearly for all protocols.

\subsection{Energy consumed}

It measures the instantaneous amount of energy being consumed in the network per round. This is simply the energy difference from the beginning till the end of a round. 


\subsection{Network residual energy}

It measures the total remaining energy of the network. It is calculated at each transmission round of the protocol.

The metrics used allow us to conclude about the stability of the network which is the time interval from the start of network operation until the death of the last sensor node. The lifetime of the network defined as the number of rounds until the last node die is simply the operational period of the network that is the period for which the network continues to provide information to the BS.

\section{RESULTS AND DISCUSSIONS}

We simulated our methodology in MATLAB [20] to verify our results. Figure 7 shows that the two attributes of the nodes have different effect on the probability of the node to be leader. First, with increase in battery level and decrease in proximity to the BS, the probability of being elected as leader increases; while with increase in the distance between node and BS, the probability of node to be elected as leader decreases. The residual energy plays a very important role in selection of leader because even if the distance is far, the node of higher energy can have higher probability to be elected. So, a node of low battery level will have a low probability obviously.

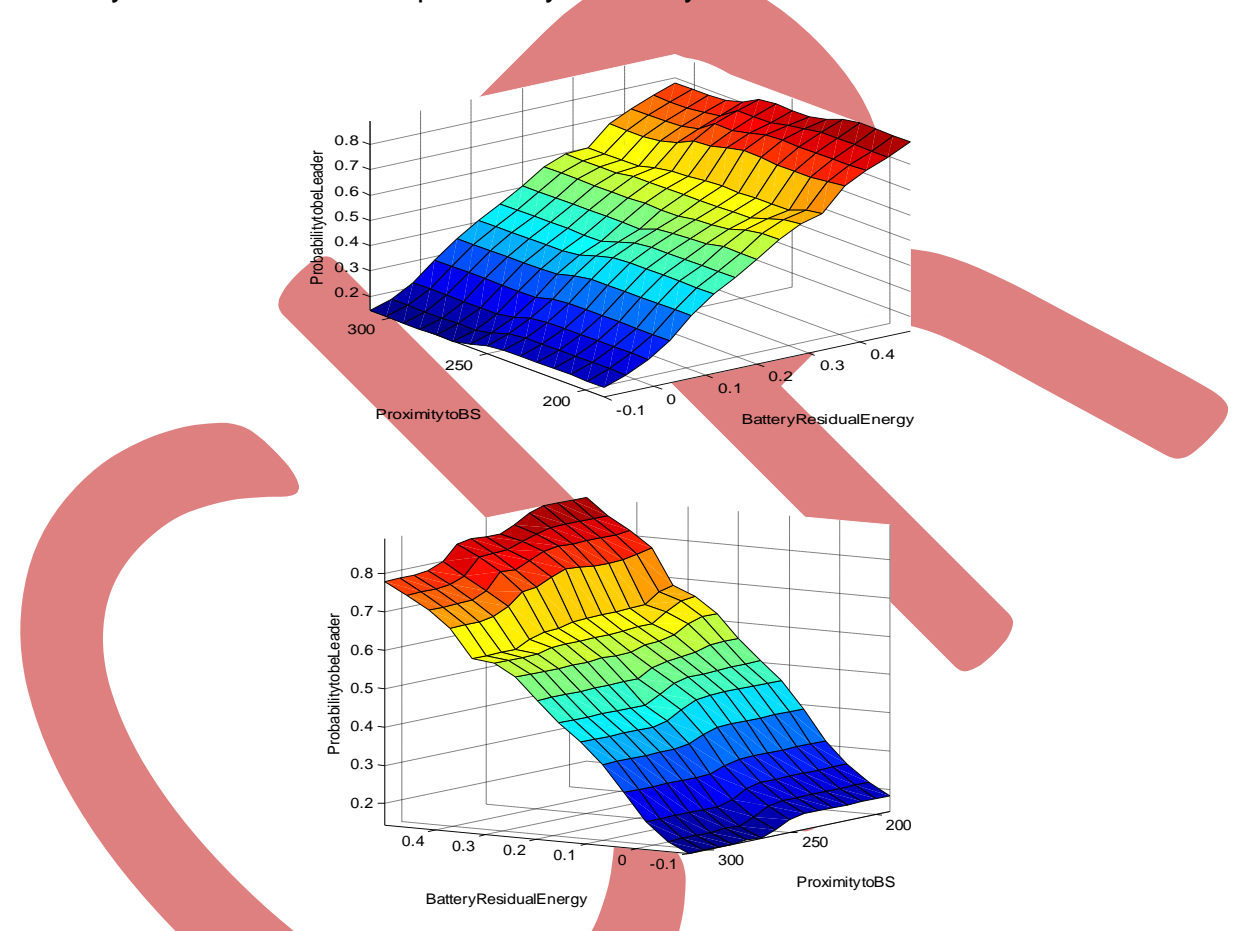

Fig 7. Surface: (a) Proximity to BS, Residual energy (b) Residual energy, proximity to BS.

To compare with PEGASIS-TC [1, 17], a network of area $100 \times 100$ meters with 100 nodes is selected. The coordinate of $\mathrm{BS}$ is $(50,300)$ and parameters are shown in table 4.

Table 4: System Parameters.

\begin{tabular}{|l|l|}
\hline Parameter & Value \\
\hline $\begin{array}{l}\text { Initial Energy of } \\
\text { Node }\end{array}$ & $0.50 \mathrm{~J}$ \\
\hline $\mathrm{E}_{\text {elect }}$ & $50 \mathrm{~nJ} / \mathrm{bit}$ \\
\hline $\mathrm{E}_{\mathrm{fs}}$ & $10 \mathrm{pJ} / \mathrm{bit} / \mathrm{m}^{2}$ \\
\hline $\mathrm{E}_{\mathrm{mp}}$ & $0.0013 \mathrm{pJ} / \mathrm{bit}^{2} \mathrm{~m}^{4}$ \\
\hline $\mathrm{E}_{\text {fusion }}$ & $5 \mathrm{~nJ} / \mathrm{bit} / \mathrm{message}$ \\
\hline Data Packet & $2000 \mathrm{bits}$ \\
\hline
\end{tabular}




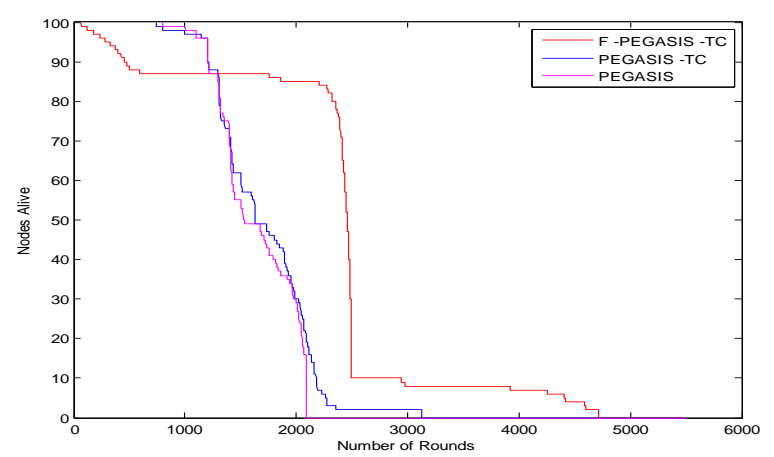

Fig 8. Number of nodes alive

Figure 8 depicts the number of alive nodes in the network. It is observed that F-PEGASIS-TC has a stable lifetime as compared to PEGASIS-TC [1, 17]. The lifetime period of F-PEGASIS-TC before the death of first node is less, but it continues to provide information about the sensor field for a longer period of time, moreover, higher number of nodes alive for a longer period of time. F-PEGASIS-TC schedules the election of leader in a more efficient manner based on residual energy and distance to BS. Often random election of leader [1, 17] leads to loss of energy from already energy deficit nodes.

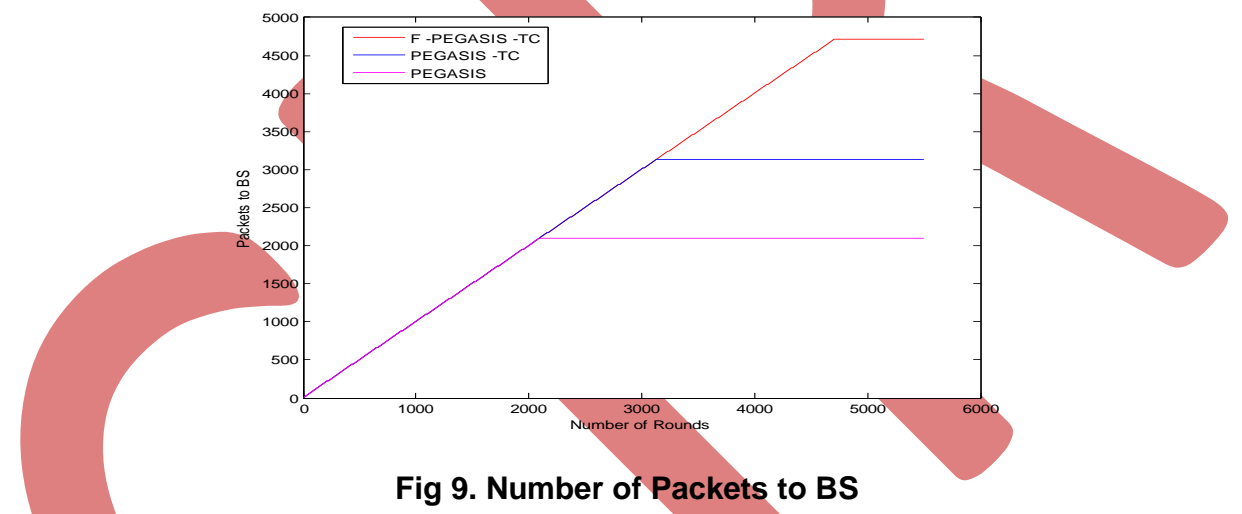

Figure 9. indicates that fuzzy only gives a gain of mere $1.25 \%$ in number of Packets sent to BS for F-PEGASIS-TC as compared to PEGASIS-TC. This is shows that lifetime is almost the same with respect to death of last node. But, Figure 7 add to point that lifetime is more substantial in F-PEGASIS-TC.

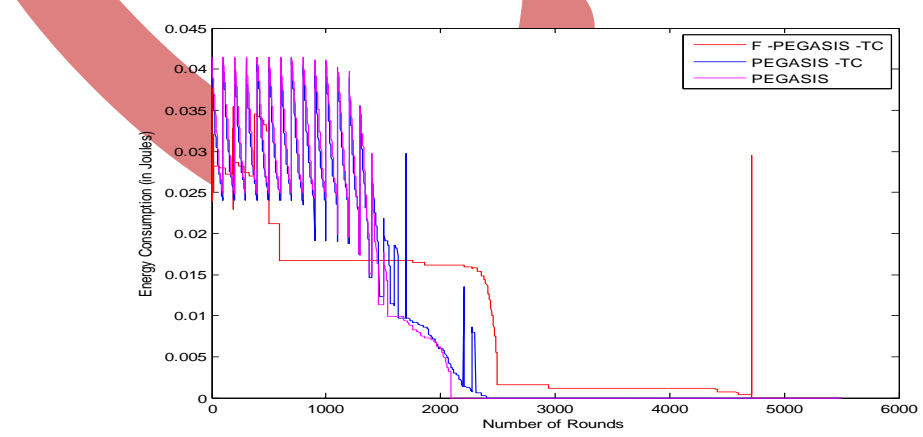

Fig 10. Energy consumed over time.

Figure 10 shows energy consumption of F-PEGASIS-TC. It is concluded that F-PEGASIS-TC consumes less energy compared to PEGASIS-TC because of smart scheduling of leader. 


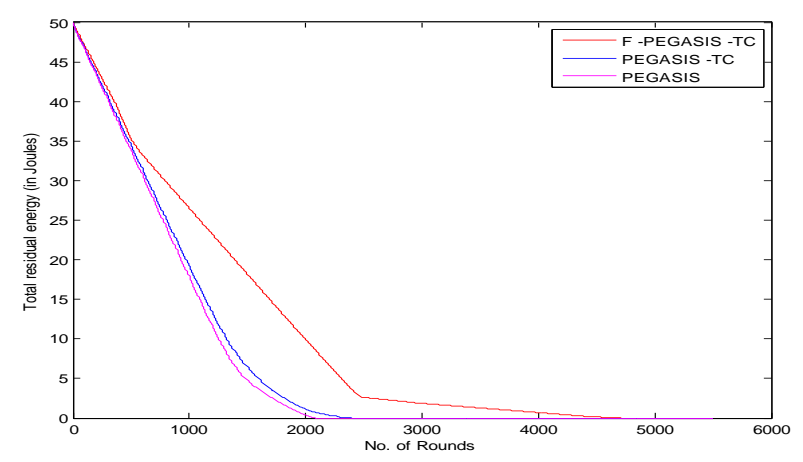

Fig 11.Total residual energy

Figure 11 depicts that F-PEGASIS-TC has more balanced energy dissipation and more stable lifetime as compared to PEGASIS-TC.

\section{CONCLUSION}

This paper proposes an improved energy-efficient PEGASIS [17] based protocol F-PEGASIS-TC, which provides energy efficient way of leader selection using Fuzzy logic and the concept of topology control for chaining [1]. The novel algorithm provides a more stable lifetime to the network. The simulation results prove that F-PEGASIS-TC outperforms PEGASISTC by achieving higher energy-efficiency extending lifetime of network.

\section{REFERENCES}

[1] Paolo Santi, "Topology Control in Wireless AdHoc and Sensor Networks", ACM Computing Surveys, Vol. 37, No. 2, June 2005, pp. 164-194.

[2] Ahmed A. Ahmed, Hongchi Shi \& Yi Shang, "A Survey on Network Protocols for Wireless Sensor Networks", IEEE 2003.

[3] I.F. Akyildiz et.al.," Wireless sensor networks: a survey." Computer Networks, 2002, 38 (4) pp:393-422.

[4] Jamal N. Al-Karaki, Ahmed E. Kamal, " Routing Techniques in Wireless Sensor Networks: A Survey", IEEE Wireless Communications, Volume: 11, Issue: 6 ,

[5] Yunxia Chen and Qing Zhao, "On the Lifetime of Wireless Sensor Networks", Communications Letters, IEEE, Volume 9, Issue 11, pp:976-978, Digital Object Identifier 10.1109/ LCOMM.2005.11010., Nov. 2005.

[6] S. Lindsey, C. S. Raghavendra and K.Sivalingam, "Data Gathering in Sensor Networks using energy delay metric", In Proceedings of the $15^{\text {th }}$ International Parallel and Distributed Processing Symposium, pp 188-200,2001.

[7] Jong-Myoung Kim, Seon-Ho Park, Young-Ju Han, Tai-Myoung Chung, "CHEF: Cluster Head Election mechanism using Fuzzy logic in Wireless Sensor networks", Feb. 17-20, 2008 ICACT 2008, ISBN 978-89-5519-136-3

[8] S. Swapna Kumar, M. Nanda Kumar, V.S. Sheeba, K.R. Kashwan, "Cluster Based Routing Algorithm using Dual Staged Fuzzy Logic in Wireless Sensor Networks", Journal of Information \& Computational Science 9: 5 (2012) 12811297

[9] I. Gupta, D. Riordan and S. Sampalli, "Cluster Head Election using Fuzzy Logic for Wireless Sensor Networks", Communication networks and Services Research conference, pp.255-260, May 2005.

[10] Hironori Ando, Leonard Barolli, Arjan Durresi, Fatos Xhafa, and Akio Koyama, "An Intelligent Fuzzy-based Cluster Head Selection System for Wireless Sensor Networks and Its Performance Evaluation", 2010 13th International Conference on Network-Based Information Systems 978-0-7695-4167-9/10 \$26.00 @ 2010 IEEE DOI 10.1109/ NBiS.2010.72

[11] Ashutosh Kumar Singh, Abhijeet Alkesh, N.Purohit, "Minimization of Energy Consumption of Wireless Sensor Networks using Fuzzy Logic", 2011 International Conference on Computational Intelligence and Communication Systems 978-0-7695-4587-5/11 \$26.00 @ 2011 IEEE DOI 10.1109/CICN.2011.111

[12] Ge Ran, Huazhong Zhang, Shulan Gong, "Improving on LEACH Protocol of Wireless Sensor Networks Using Fuzzy Logic", Journal of Information \& Computational Science 7: 3 (2010) 767-775 1548-7741/ Copyright@ 2010 Binary Information Press March 2010

[13] Yu Hu, Xiaorui Shen, Zhenhua Kang, "Energy-Efficient Cluster Head Selection in Clustering Routing for Wireless Sensor Networks", 978-1-4244-3693-4/09/\$25.00 @2009 IEEE

[14] Slim Hammadi, Christian Tahon, Prof. P. Borne, "Special Issue on Intelligent Techniques in Flexible Manufacturing Systems", IEEE Transactions on systems, Man, and Cybernetics- Part C: Applications and Reviews, Vol. 33, No. 2, May 2003 
[15] W. Heinzelman, A. Chandrakasan, and H. Balakrishnan, "Energy-efficient communication protocols for wireless microsensor networks," Proceedings of the 33rd International Conference on System Sciences (HICS '00), January 2000.

[16] W. Heinzelman, A. Chandrakasan, and H. Balakrishnan, "An application-specific protocol architecture for wireless microsensor networks", IEEE Transactions on Wireless Communications, Vol. 1, No. 4, pp. 660-670, October 2002.

[17] S. Lindsey, C.S. Raghavendra," PEGASIS: Power-efficient gathering in sensor information systems", IEEE Aerospace and Electronic Systems Society, Proc of the IEEE Aerospace Conf, Montana, 2002:1125-1130.

[18] Feng Sen, Qi Bing, Tang Liangrui, "An Improved Energy Efficient PEGASIS Based Protocol in Wireless Sensor Networks", IEEE 2011 Eighth International Conference on Fuzzy Systems and Knowledge Discovery (FSKD).

[19] http:// www.dma.fi.upm.es

[20] http://www.mathworks.com/ Fuzzy Logic Toolbox user's guide.

[21] J. Anno, L. Barolli, A. Durresi, "A Cluster Head Selection Method for Wireless sensor Networks Based on Fuzzy Logic”, Proc. Of IEEE TENCON- 2007, CD-ROM, 4 pages, 2007.

\section{Author' biography with Photo}

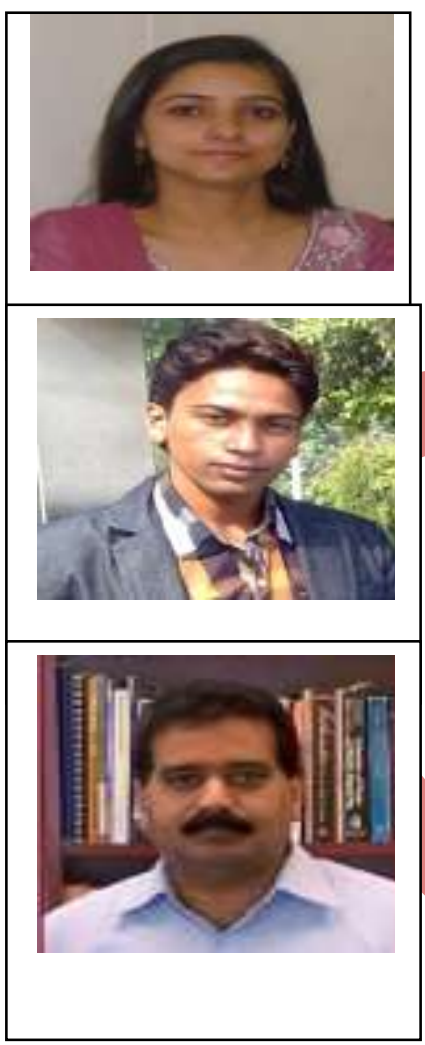

Vibha Nehra

Vibha Nehra obtained her M.Tech. in Department of Computer Science \& Engineering from Dr.B.R . Ambedkar National Institute of Technology, Jalandhar. She got her Bachelor's degree in Computer Science \& Engg. from H.R. Institute of Technology, Ghaziabad, affiliated to Uttar Pradesh Technical University, Lucknow. Her research areas are Wireless Sensor Networks, Wireless \& Adhoc Network along with cryptography and Network Security.

$$
\text { Mr. Raju Pal }
$$

Raju pal obtained M.Tech. in Computer Science \& Engineering from Dr. B. R. Ambedkar National Institute of Technology, Jalandhar (NITJ). He obtained his B.Tech. in Information Technology from Babu Banarasi Das Institute of Technology, Ghaziabad affiliated by Uttar Pardesh Technical University, Lucknow. His area of research is Wireless Sensor Networks, Wireless and Ad-Hoc Network and Network Security.

\section{Dr Ajay K Sharma}

Ajay K Sharma received his BE in Electronics and Electrical Communication Engineering from Punjab University Chandigarh, India in 1986, MS in Electronics and Control from Birla Institute of Technology (BITS), Pilani in the year 1994 and PhD in Electronics Communication and Computer Engineering in the year 1999. His PhD thesis was on "Studies on Broadband Optical Communication Systems and Networks". From 1986 to 1995 he worked with TTTI, DTE Chandigarh, Indian Railways New Delhi, SLIET Longowal and National Institute of technology (Erstwhile Regional Engineering College), Hamirpur HP at various academic and administrative positions. He has joined National Institute of Technology (Erstwhile Regional Engineering College) Jalandhar as Assistant Professor in the Department of Electronics and Communication Engineering in the year 1996. From November 2001, he has worked as Professor in the ECE department and presently he working as Professor in Computer Science \& Engineering in the same institute. His major areas of interest are broadband optical wireless communication systems and networks, dispersion compensation, fiber nonlinearities, optical soliton transmission, WDM systems and networks, Radio-over-Fiber (RoF) and wireless sensor networks and computer communication. 